\title{
Comparison of Wide Awake Local Anaesthesia No Tourniquet Technique with Tourniquet Application under General Anesthesia in Carpal Tunnel Syndrome: A Retrospective Study
}

(D) Mustafa Çağlar Kır, (D) Mehmet Kürşad Bayraktar, (D) Hakan Gürbüz

Istanbul Okmeydanı Training and Research Hospital, Clinic of Orthopaedics and Traumatology, Istanbul, Turkey

\section{Abstract}

Objective: Carpal tunnel syndrome is a common complaint in orthopedic practice and usually treated with surgical intervention. The aim of the study was to compare wide awake local anesthesia no tourniquet (WALANT) technique with general anesthesia and arm tourniquet in Carpal tunnel cases.

Methods: Fifty-two patients who underwent surgery for Carpal tunnel syndrome were retrospectively divided into two groups as WALANT technique (Group W) and general anesthesia with arm tourniquet (Group G). In addition to the demographic data, visual analog scale (VAS), opioid consumption, early complications, duration of stay in operating room (DSOR) and first analgesic need time were evaluated.

Results: When both groups were evaluated, early postoperative VAS scores, opioid consumption and DSOR were significantly lower in Group W than in Group G.

Conclusion: In conclusion, WALANT technique was associated with reduced early postoperative pain, lower DSOR and opioid consumption compared to general anesthesia with tourniquet method for carpal tunnel release.

Keywords: Wide awake local anesthesia no tourniquet, Carpal tunnel syndrome, general anesthesia, pain

\section{INTRODUCTION}

Carpal tunnel syndrome, which affects $3 \%$ of the population, is an orthopedic condition that often requires surgical approach for permanent treatment (1). In fact, Carpal tunnel surgery is one of the most common surgical wrist procedures in the United States with 300,000 to 600,000 cases per year (2). Anesthesia and surgical techniques may vary according to surgeon preferences and patient-related factors (3). Despite the increasing popularity of local anesthetic methods, general and regional anesthesia techniques are still widely used (4). While elderly patients prefer local methods, younger patients often prefer general anesthesia (5). Adverse effects and complications may occur up to $25 \%$ of patients after Carpal tunnel surgery $(5,6)$. Problems that may develop during the surgery or in the very first 24 hours include pain, airway obstruction, bleeding, difficulty in awakening, hypoxia, hypotension, hypertension, nausea and vomiting
(5). These all could increase morbidity and reduce patient satisfaction. Tourniquet devices are commonly used in extremity surgery to reduce blood loss and create a blood-free surgical site (7). Though well improved so far, tourniquet techniques have been still complicated with pain, paresthesia, permanent and transient nerve injury, compartment syndrome, lymphedema, and arteriovenous shunt, etc. $(8,9)$. After the misconception that low epinephrine concentrations lead to permanent vasospasm and necrosis in the digits was falsified, wide awake local anesthesia no tourniquet (WALANT) anesthesia technique was started to be applied as it minimizes bleeding without using tourniquet and sedation and eases the surgical intervention (10). Other advantages of a such wide-awake anesthesia technique include no need for perioperative monitoring, early ambulation, reduced costs and the ability to cooperate with the patient for active movement examination during tendon, fracture and ligament procedures $(11,12)$. 
This study aimed to compare WALANT technique with tourniquet application under general anesthesia in terms of postoperative complications and function outcomes in cases that underwent open release due to Carpal tunnel syndrome.

\section{METHODS}

The study was conducted per ethical standards of the 1964 Helsinki Declaration. Medical records of 93 cases that underwent open release due to Carpal tunnel syndrome between January 2015 and January 2017 was retrospectively reviewed. A total of 41 cases were excluded due to reasons such as revision Carpal tunnel surgery, previous hand-wrist surgery, history of upper extremity malignancy, vascular insufficiency, only undergoing local anesthesia or regional blockade, and missing medical data or regular follow-up. The remaining 52 cases were classified by their applied anesthesia technique as Group W (WALANT anesthesia, $n=23$ ) and Group $\mathrm{G}$ (general anesthesia plus forearm tourniquet, $n=29$ ). All cases in Group $G$ were administered propofol 2-3 mg/ $/ \mathrm{kg}$, fentanyl $2-5 \mu \mathrm{g} / \mathrm{kg}$, midazolam $0.2 \mathrm{mg} / \mathrm{kg}$ and rocuronium $0.6 \mathrm{mg} / \mathrm{kg}$ for inducing anesthesia, which was afterwards maintained by $\mathrm{N}_{2} \mathrm{O}$ /sevoflurane via laryngeal mask. After a 3 minute arm elevation, an $8 \mathrm{~cm}$ forearm tourniquet supported by adequate cotton was applied such that systolic blood pressure was kept above $100 \mathrm{mmHg}$. Subjects in Group W received a $10 \mathrm{~mL}$ subcutaneous injection (27G needle) consisting of $1 \%$ lidocaine, $8.4 \%$ bicarbonate and buffered 1/100,000 epinephrine, without the use of any sedation or tourniquet. A 2-3 cm palmar longitudinal incision was made between thenar and hypothenar eminence along the axis of the $4^{\text {th }}$ digit. The skin and subcutaneous tissue were passed with sharp dissection, and transverse carpal ligament was thoroughly released with no.: 15 scalpel blade (13). All cases were applied elevation on the first day. Active finger movements were allowed as much as tolerated after two days. The subjects were followed with dressing and elastic bandage until removal of sutures at the end of two weeks, after which they were allowed to resume daily working routine.

Both groups received intravenous paracetamol $15 \mathrm{mg} / \mathrm{kg}$ every 8 hours for postoperative analgesia. In case of severe pain [visual analog scale (VAS) >3], tramadol $2 \mathrm{mg} / \mathrm{kg}$ was added to the analgesic regimen. Demographic characteristics, early postoperative complications (nausea, vomiting, airway problem, bleeding, hypotension, hypertension, paresthesia, nerve injury), VAS score at hour 24 and 72, duration of stay in operating room (DSOR), postoperative opioid consumption and first analgesic need time (FANT) were recorded.

\section{Statistical Analysis}

IBM SPSS Statistics 22 program was used for statistical analysis. The distribution of the data was evaluated by KolmogorovSmirnov test. Descriptive statistical methods (mean, standard deviation) were used in the analysis. Independent Samples t-test was utilized for parametric values. Fisher exact test was used for qualitative data. $\mathrm{P}<0.05$ was assessed as statistically significant.

\section{RESULTS}

The mean age of the patients was similar between Group $\mathrm{W}$ (63.7 \pm 6.1 years) and Group G $(62.7 \pm 6.2$ years) $(p=0.53)$. Female patients constituted the majority of cases in both groups, Group W $(70 \%, \mathrm{n}=16)$ and Group $\mathrm{G}(65.5 \%, \mathrm{n}=19)$. Although the groups did not significantly differ in terms of operative time [Group W=17.26 \pm 4.90 minute $(\mathrm{min})$, Group $\mathrm{G}=17.04 \pm 5.11 \mathrm{~min}$; $\mathrm{p}=0.87]$, DSOR was significantly higher in Group G compared to that of Group W $(46.48 \pm 5.49$ min vs. $33.43 \pm 6.27 \mathrm{~min}$, respectively; $p=0.0001$ ) (Table 1 ). Analysis of early postoperative pain showed that 24-hour and 72-hour VAS scores were significantly lower in Group W compared to those in Group G (24hour VAS $=3.13 \pm 1.79$ vs. $4.55 \pm 1.62$, respectively; $p_{24}=0.0042$ and 72-hour VAS $=2.26 \pm 0.92$ vs. $3.59 \pm 0.87$, respectively; $\mathrm{p}_{72}=0.0001$ ). FANT was longer in Group W than in Group G $(282 \pm 68.27 \mathrm{~min}$ vs. $121.03 \pm 31.01 \mathrm{~min}$, respectively; $p=0.0001$ ). The opioid consumption due to severe pain was found to be more common

\begin{tabular}{|c|c|c|c|}
\hline & $\begin{array}{l}\text { Group W } \\
(\text { Mean } \pm \text { SD) }\end{array}$ & $\begin{array}{l}\text { Group G } \\
\text { (Mean } \pm \text { SD) }\end{array}$ & $p$ \\
\hline Age, years & $63.74 \pm 6.08$ & $62.66 \pm 6.23$ & 0.53 \\
\hline OT, minutes & $17.26 \pm 4.90$ & $17.04 \pm 5.11$ & 0.87 \\
\hline DSOR, minutes & $33.43 \pm 6.27$ & $46.48 \pm 5.49$ & $0.0001^{*}$ \\
\hline \multicolumn{4}{|l|}{ VAS } \\
\hline (24-hour) & $3.13 \pm 1.79$ & $4.55 \pm 1.62$ & $0.0042 *$ \\
\hline VAS & & & \\
\hline (72-hour) & $2.26 \pm 0.92$ & $3.59 \pm 0.87$ & $0.0001^{*}$ \\
\hline FANT, minutes & $282 \pm 68.27$ & $121.03 \pm 31.01$ & $0.0001 *$ \\
\hline
\end{tabular}


in Group G than that in Group W ( $27 \%$ vs. $4.3 \%$, respectively; $p=0.036)$ (Table 2).

While two cases (8.6\%) developed transient paresthesia in Group W, a total of six cases (20.6\%) in Group G experienced early postoperative complications, including two cases with paresthesia, and others with nausea, vomiting, hypertension, and postoperative spasm. The difference in complication rates was not statistically significant $(p=0.27)$.

Table 2. Evaluation of early postoperative complication and opioid consumption rate for Group W and Group G

\begin{tabular}{|c|c|c|c|c|c|}
\hline \multirow{3}{*}{$\begin{array}{l}\text { Opioid } \\
\text { consumption }\end{array}$} & \multicolumn{2}{|c|}{ Group W } & \multicolumn{2}{|c|}{ Group G } & \multirow{2}{*}{$p$} \\
\hline & $n$ & $\%$ & $\mathrm{n}$ & $\%$ & \\
\hline & 1 & 4.3 & 8 & 27 & $0.036^{*}$ \\
\hline $\begin{array}{l}\text { Early } \\
\text { complication }\end{array}$ & 2 & 8.6 & 6 & 20.6 & 0.27 \\
\hline
\end{tabular}

\section{DISCUSSION}

The most important finding of this study is the superior efficacy of WALANT anesthesia in early postoperative pain over general anesthesia plus forearm tourniquet in cases undergoing Carpal tunnel release procedure. Literature search did not show any direct comparison regarding these modalities. Nevertheless, Iqbal et al. (14) published a study in 2018 in which they randomized Carpal tunnel release cases to undergo either local anesthesia plus adrenaline or local anesthesia plus tourniquet and they found that early pain score was significantly higher in the latter group. Gunasagaran et al. (15) compared WALANT technique to local anesthesia plus tourniquet in 72 cases with minor hand surgery and they reported lower early postoperative pain in subjects undergoing WALANT technique. Consistent with the literature, our findings may be attributed the prolongation of postoperative analgesic time with adrenaline and/or aggravation of postoperative pain level by tourniquet application. In a study consisting of 108 cases, Gibson (16) reported that addition of adrenalin to lidocaine was associated with increased postoperative analgesic time at the expense of relatively low complication rates. Consistent to that, we also detected WALANT technique to prolong the FANT. It may be speculated that epinephrine-induced vasoconstriction in tissues may extend the duration of action of local anesthetic agent. WALANT technique is becoming increasingly popular in hand and wrist surgery. Compared with other anesthesia methods, it is reported as a usually reliable, cost-effective and timesaving procedure (17-19). Other advantages of the technique include avoidance of tourniquet-related pain and other complications and evaluation of perioperative active movements via patient cooperation. Finger fracture fixation, tendon repair or transfer, arthrodesis, arthroplasty, release of median nerve and trigger finger could be performed with this technique (15). In this study, we performed open median nerve release with this technique. Though the duration of the surgery did not alter, DSOR was shorter than that in general anesthesia and tourniquet technique. Carpal tunnel release is one of the most common interventions in hand surgery practice. Despite being a minor surgery, it was reported to be complicated in up to one-fourth of cases. In our study, $20.6 \%$ of general anesthesia plus tourniquet group had early postoperative complications, including paresthesia, nausea, vomiting, hypertension, and bronchospasm. On the other hand, it was remarkable that $8.6 \%$ of WALANT group had transient paresthesia. The difference between the groups was not significant. Rozanski et al. (5) compared general, regional and local anesthesia in patients undergoing median nerve release and reported that early postoperative complications were found to be more common in local anesthesia than that in general anesthesia. The conflict with our study may be related with the addition of epinephrine to local anesthetic, varying general and local anesthetic techniques, and different levels of preoperative patient counseling about the conditions that may be encountered. Opioids are frequently used for the management of severe postoperative pain. In fact, the use of these drugs has increased in recent years. A study in the United States reported $142 \%$ increment of opioid prescriptions in the emergency setting and $42 \%$ increment of total opioid use from 2002 to 2009 (20,21). Chapman et al. (20) reported no difference in opioid consumption between WALANT technique and sedation in the study they performed on 277 cases with Carpal tunnel syndrome. In our study, opioid use was found to be less in the WALANT group than in patients undergoing general anesthesia plus tourniquet. It might be partially attributed to be able to establish a better postoperative analgesia with WALANT technique. Our study has several limitations, including a singlecenter retrospective design, small study sample, and absence of mid- and long-term outcomes and follow-up of perioperative hemodynamic parameters.

\section{CONCLUSION}

In conclusion, WALANT technique was associated with reduced early postoperative pain, opioid consumption and DSOR compared to that in general anesthesia plus tourniquet method in cases who underwent open median nerve release. 


\section{Ethics}

Ethics Committee Approval: Local Editorial Board approval was received from Okmeydanı Training and Research Hospital, Department of Orthopaedic and Traumatology (8060875-E 18634).

Informed Consent: Was obtained.

Peer-review: Externally peer-reviewed.

\section{Authorship Contributions}

Surgical and Medical Practices: M.Ç.K., M.K.B., H.G., Concept: M.Ç.K., M.K.B., H.G., Design: M.C..K., M.K.B., Data Collection or Processing: M.C..K., Analysis or Interpretation: M.C..K., Literature Search: M.C..K., M.K.B., Writing: M.Ç.K., M.K.B., H.G .

Conflict of Interest: No conflict of interest was declared by the authors.

Financial Disclosure: The authors declared that this study received no financial support.

\section{REFERENCES}

1. Concannon MJ, Brownfield ML, Puckett CL. The incidence of recurrence after endoscopic carpal tunnel release. Plast Reconstr Surg 2000;105:1662-5.

2. Fajardo M, Kim SH, Szabo RM. Incidence of Carpal Tunnel Release: Trends and Implications Within the United States Ambulatory Care Setting. J Hand Surg Am 2012;37:1599-605.

3. Michelotti B, Romanowsky D, Hauck RM. Prospective, randomized evaluation of endoscopic versus open carpal tunnel release in bilateral carpal tunnel syndrome: an interim analysis. Ann Plast Surg 2014;73:157-60.

4. Foster BD, Sivasundaram L, Heckmann N, Cohen JR, Pannell WC, Wang JC, et al. Surgical Approach and Anesthetic Modality for Carpal Tunnel Release. Hand 2017;12:162-7.

5. Rozanski M, Neuhaus V, Thornton E, Becker SJ, Rathmell JP, Ring D. Symptoms During or Shortly After Isolated Carpal Tunnel Release and Problems Within 24 hours After Surgery. J Hand Microsurg 2015;7:30-5.

6. Stutz N, Gohritz A, van Schoonhoven J, Lanz U. Revision surgery after carpal tunnel release--analysis of the pathology in 200 cases during a 2 year period. J Hand Surg Br 2006;31:68-71.
7. Drosos Gl, Ververidis A, Stavropoulos NI Mavropoulos R, Tripsianis G, Kazakos K. Silicone ring tourniquet versus pneumatic cuff tourniquet in carpal tunnel release: A randomized comparative study. J Orthop Traumatol 2013;14:131-5.

8. Odinsson A, Finsen V. Tourniquet use and its complications in Norway. J Bone Jt Surg - Br Vol 2006;88:1090-2.

9. Burk RW, Guzman-Stein G, Vasconez LO. Lidocaine and epinephrine levels in tumescent technique liposuction. Plast Reconstr Surg 1996;97:1379-84.

10. Lalonde D, Bell M, Benoit P, Sparkes G, Denkler K, Chang P. A multicenter prospective study of 3,110 consecutive cases of elective epinephrine use in the fingers and hand: The dalhousie project clinical phase. J Hand Surg Am 2005;30:1061-7.

11. Lalonde D. Conceptual origins, current practice, and views of wide awake hand surgery. J Hand Surg Eur Vol 2017;42:886-95.

12. Farhangkhoee $\mathrm{H}$, Lalonde J, Lalonde $\mathrm{DH}$. Teaching medical students and residents how to inject local anesthesia almost painlessly. Can J Plast Surg 2012;20:169-72

13. Tulipan J, Kim N, Abboudi J Jones C, Liss F, Kirkpatrick W, et al. Open Carpal Tunnel Release Outcomes: Performed Wide Awake versus with Sedation. J Hand Microsurg 2017;9:74-9.

14. Iqbal HJ, Doorgakant A, Rehmatullah NNT, Ramavath AL, Pidikiti P, Lipscombe S. Pain and Outcomes of Carpal Tunnel Release Under Local Anaesthetic with or without a Tourniquet: A Randomized Controlled Trial. J Hand Surg Eur Vol 2018;43:808-12.

15. Gunasagaran J, Sean ES, Shivdas S, Amir S, Ahmad TS. Perceived comfort during minor hand surgeries with wide awake local anaesthesia no tourniquet (WALANT) versus local anaesthesia (LA)/tourniquet. J Orthop Surg (Hong Kong) 2017;25:2309499017739499.

16. Gibson M. Outpatient carpal tunnel decompression without tourniquet: a simple local anaesthetic technique. Ann R Coll Surg Engl 1990;72:408-9.

17. McKee DE, Lalonde DH, Thoma A, Dickson L. Achieving the optimal epinephrine effect in wide awake hand surgery using local anesthesia without a tourniquet. Hand 2015;10:613-5.

18. Codding JL, Bhat SB and Ilyas AM. An economic analysis of MAC versus WALANT: a trigger finger release surgery case study. Hand 2016;12:348-51.

19. Al Youha S, Lalonde DH. Update/review: changing of use of local anesthesia in the hand. Plast Reconstr Surg Glob Open 2014;6:e150.

20. Chapman T, Kim N, Maltenfort M, Ilyas AM. Prospective Evaluation of Opioid Consumption Following Carpal Tunnel Release Surgery. Hand 2017;12:3942.

21. Volkow ND, McLellan TA, Cotto JH, Karithanom M, Weiss S. Characteristics of opioid prescriptions in 2009. JAMA 2011;305:1299-301. 